\title{
Quantum Kinetic Transport under High Electric Fields
}

\author{
NOBUYUKI SANO ${ }^{*}+\dot{*}$ and AKIRA YOSHII \\ NTT LSI Laboratories, 3-1 Morinosato Wakamiya, Atsugi-shi, Kanagawa 243-01, Japan
}

\begin{abstract}
Quantum kinetic transport under high electric fields is investigated with emphasis on the intracollisional field effect (ICFE) in low-dimensional structures. It is shown that the ICFE in $\mathrm{GaAs}$ one-dimensional quantum wires is already significant under moderate electric field strengths ( $\geq$ a few hundreds $\mathrm{V} / \mathrm{cm}$ ). This is a marked contrast to the cases in bulk, where the ICFE is expected to be significant under extremely strong electric fields ( $\geq \mathrm{MV} / \mathrm{cm})$. Employing the Monte Carlo method including the ICFE, the electron drift velocity in quantum wires is shown to be much smaller than that expected from earlier investigations.
\end{abstract}

Keywords: quantum transport, hot electron, monte carlo, quantum wire, intracollisional field effect, collisional broadening

\section{INTRODUCTION}

The fast development of semiconductor devices has brought about renewed interest in high-field carrier transport in semiconductors. Among others, the breakdown of the semiclassical Boltzmann transport equation under high electric fields, on which most conventional analyses of carrier transport in semiconductor devices are based, has been widely predicted.[1] However, the predicted electric field strengths under which the semiclassical treatment of carrier transport is supposed to break down vary from several tens $\mathrm{kV} / \mathrm{cm}$ to $\mathrm{MV} / \mathrm{cm}$. Therefore, our understanding of quantum carrier transport under high electric fields is still immature. In the present paper, we investigate the quantum effects in electron transport from a rather different point of view; we apply the quantum kinetic transport equation (the Barker-Ferry equation [2]) to low-dimensional structures so that the quantum effects are, in some cases, more pronounced than in the three-dimensional (3-D) bulk cases and their physical meanings become more transparent.

We consider ideal 1-D GaAs quantum wires and investigate the intracollisional field effect (ICFE) on electron transport via the Monte Carlo method.[3] To the best of our knowledge, this is the first time the ICFE on high-temperature electron transport in lowdimensional structures has been taken into account. Since the ICFE is mainly ascribed to the energy change of an electron by the electric field during the collision duration with phonons, [2] it is expected that the ICFE could be most significant when the electron motion is restricted to the dimension parallel or antiparallel to the electric field. As we shall show, this is indeed the case in 1-D quantum wire structures; the ICFE becomes significant even under moderate electric field strengths and greatly affects transport characteristics such as the drift velocity.

* Corresponding author. e-mail: sano@aecl.ntt.jp, phone: +81-462-40-2616, FAX: +81-462-40-4306

$\dagger$ Present address: Institute of Applied Physics, University of Tsukuba, Tsukuba, Ibaraki 305, Japan 


\section{QUANTUM KINETIC TRANSPORT EQUATION}

Our starting point is the quantum kinetic transport equation (the Barker-Ferry equation) for the one-particle distribution function of electrons $f_{\mathbf{k}}$, which is derived from the quantum Liouville equation for the reduced electron density matrix.[4] Under a constant electric field $\mathbf{F}$ with the non-degenerate condition, the Barker-Ferry equation for electrons is given by

$$
\begin{aligned}
& \frac{\partial f_{\mathbf{k}}(t)}{\partial t}-e \mathbf{F} \cdot \frac{\partial f_{\mathbf{k}}(t)}{\partial \mathbf{k}}= \\
& \quad 2 \operatorname{Re} \sum_{\mathbf{q}, \eta= \pm} \int_{0}^{t} d \tau\left|M_{\mathbf{q}}\right|^{2}\left(N_{\mathbf{q}}+\frac{1}{2}+\frac{\eta}{2}\right) \\
& \left\{e^{-i\left(\varepsilon_{\mathbf{k}_{F}+\eta \mathbf{q}}-\varepsilon_{\mathbf{k}_{F}}-\eta \omega_{\mathbf{q}}\right) \tau} f_{\mathbf{k}_{F}-\mathbf{q}}(t-\tau)\right. \\
& \left.-e^{-i\left(\varepsilon_{\mathbf{k}_{F}-\eta \mathbf{q}}-\varepsilon_{\mathbf{k}_{F}}+\eta \omega_{\mathbf{q}}\right) \tau} f_{\mathbf{k}_{F}}(t-\tau)\right\},
\end{aligned}
$$

with $\mathbf{k}_{F}=\mathbf{k}-\mathrm{e} \mathbf{F} \tau$. Here, $\varepsilon_{\mathbf{k}}$ is the electron energy with wave-vector $\mathbf{k}, \mathbf{q}$ the phonon wave-vector, $M_{\mathbf{q}}$ the matrix element for the electron-phonon interaction, $\omega_{\mathbf{q}}$ the longitudinal optical (LO) phonon energy, and $N_{\mathrm{q}}$ the phonon occupation number given by the BoseEinstein statistics. $\eta=1(-1)$ for phonon emission (absorption). Note that we use the units of $\hbar=1$ throughout the paper.

Notice that Eq. (1) is very similar in form to the semiclassical Boltzmann transport equation except for the collision integral [the right-hand side of Eq. (1)]; i.e., the time retardation of the collision dynamics with phonons is taken into account. Because of this time retardation, a numerical evaluation of Eq. (1) is still a formidable task. Since the electron energy distribution usually spreads over the wide energy ranges under high electric fields, the time retardation of the electron distribution function in the collision integral may be safely ignored. As a result, the collision integral $I_{c}$ in Eq. (1) is rewritten as

$$
\begin{aligned}
I_{c}=2 \pi \sum_{\mathbf{q}, \eta= \pm}\left|M_{\mathbf{q}}\right|^{2}\left(N_{\mathbf{q}}+\frac{1}{2}+\frac{\eta}{2}\right) \\
\left\{S\left(\eta, \mathbf{k}_{F}\right) f_{\mathbf{k}-\mathbf{q}}(t)-S\left(-\eta, \mathbf{k}_{F}\right) f_{\mathbf{k}}(t)\right\},
\end{aligned}
$$

where the spectral density $S\left(\eta, \mathbf{k}_{F}\right)$ is given by

$$
S\left(\boldsymbol{\eta}, \mathbf{k}_{F}\right)=\frac{1}{\pi} \int_{0}^{\infty} d \tau e^{-\Gamma_{\mathbf{k}} \tau} \cos \left[\left(\varepsilon_{\mathbf{k}_{F}+\eta \mathbf{q}}-\varepsilon_{\mathbf{k}_{F}}-\eta \omega_{\mathbf{q}}\right) \tau\right] \text {. }
$$

Here, $\Gamma_{\mathbf{k}}$ is the total phonon scattering rate and should be determined self-consistently with Eq. (1). Recall that Eq. (1) is derived under the Born approximation,[4] in which only the lowest-order correction for the electron-phonon interaction is included. Therefore, we put damping factor $\Gamma_{k}$ in the collision integral and extend the upper limit of the time integral to infinity because the time correlation would be destroyed by a consecutive collision with phonons. When the electron lifetime is infinite $\left(\Gamma_{k}=0\right)$ and the electric field is suppressed ( $F=0$ ), Eq. (3) correctly reduces to the energy-conserving delta function and Eq. (1) with Eq. (2) becomes identical to the Boltzmann transport equation. Fortunately, Eq. (3) can be analytically solved for a fixed $\Gamma_{\mathbf{k}}$ and plotted with solid lines under $F=500 \mathrm{~V} / \mathrm{cm}$ in Fig. 1. The electric field is assumed to be directed to the negative direction. Notice that the direction to which the spectral density is skewed is dependent on the direction of the electron motion; when the electron propagates against the electric field [Fig. 1 (a)], the spectral density shifts
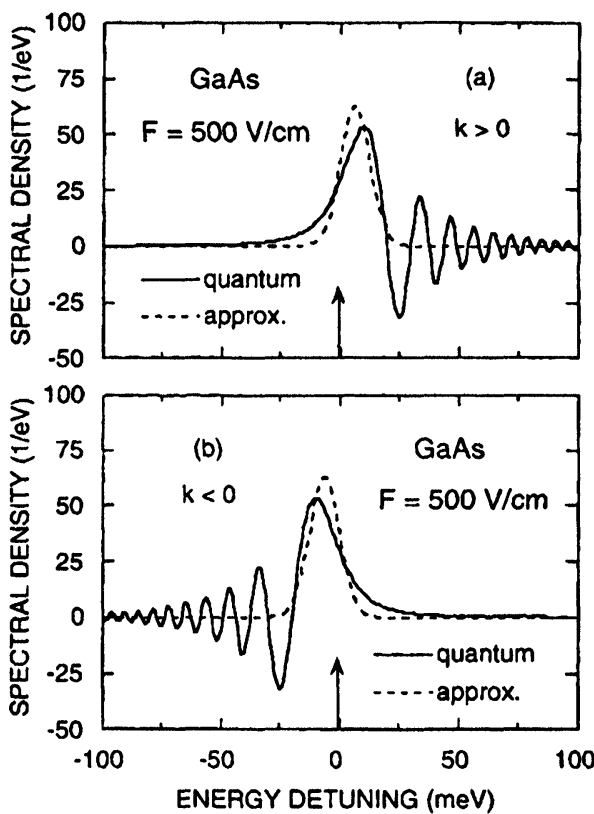

FIGURE 1 Electron spectral density as a function of energy detuning for the electrons propagating to the (a) positive and (b) negative directions under $F=500 \mathrm{~V} / \mathrm{cm}$ (solid lines). The dashed lines represent the approximated spectral densities employed in the present Monte Carlo simulations 
to the positive direction, and vice versa. This will be explained in the next section along with the phonon scattering rates for quantum wires.

It is clear from Fig. 1 that the ICFE essentially consists of two different effects: (1) the energy-conserving delta function is broadened and (2) the energy detuning (zero-point) is shifted. Both effects are directly related to the strength and the direction of the electric field, and the magnitude of both the broadening and the shift, $\Delta_{F}$, is approximately given by

$$
\Delta_{F} \sim\left(\frac{e|\mathbf{q} \cdot \mathbf{F}|}{2 m^{*}}\right)^{\frac{1}{2}}
$$

where $m^{*}$ is the electron effective mass. We would like to stress that $\Delta_{F}$ is dependent of the direction of the phonon wave-vector, or equivalently, of the electron motion. Since the electron motion is strictly confined along the electric field in 1-D quantum wires, $\Delta_{F}$ is always finite and, thus, the ICFE is always effective. On the other hand, the ICFE is not always significant in 3-D bulk because electrons can move in any direction.

\section{QUANTUM TRANSPORT IN 1-D QUANTUM WIRES}

The quantum kinetic equation described in the previous section can be easily applied to ideal 1-D quantum wires in GaAs. For simplicity, we assume that the rectangular quantum wire with the size $30 \mathrm{~nm}$ is formed by an infinitely deep potential well and only the lowest subband is occupied. In addition, only the Fröhlich optical phonon scattering in bulk mode is considered. Since the emphasis of the present analyses is on how the dynamical quantum effects affect electron transport in 1-D quantum wires, the details of the scattering modes related to the low-dimensional structure itself essentially do not change the present results.

Figure 2 shows the Fröhlich scattering rates (solid and dashed lines) obtained from the spectral density Eq. (3) under two different field strengths; $F=100$ and $500 \mathrm{~V} / \mathrm{cm}$. As well known from earlier studies on the collisional broadening (CB),[6] the divergence of the scattering rate, indicated by dotted lines in Fig. 2,
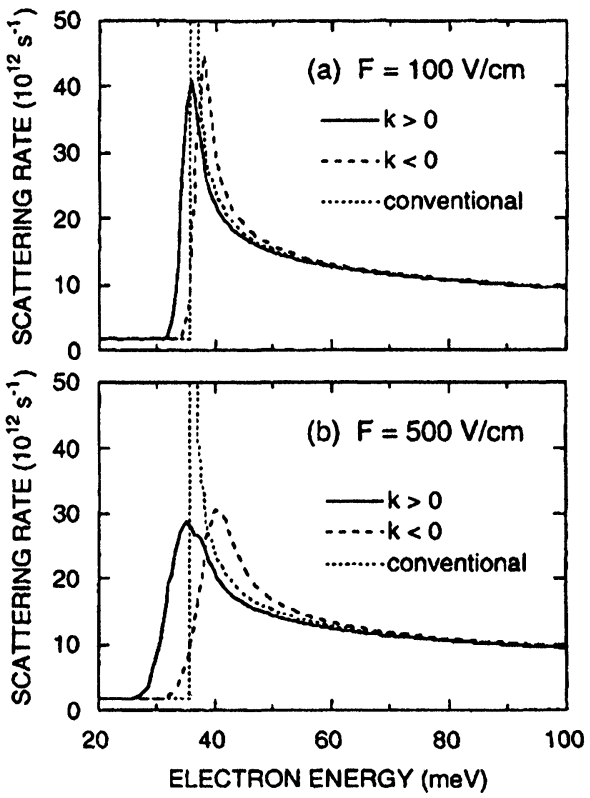

FIGURE 2 Energy dependence of the Fröhlich optical phonon scattering rates at room temperature under $F=$ (a) $100 \mathrm{~V} / \mathrm{cm}$ and (b) $500 \mathrm{~V} / \mathrm{cm}$. The solid and dashed lines represent the scattering rates for the electrons propagating to the positive and negative directions, respectively. The dotted lines show the scattering rates evaluated by the Fermi golden rule

is associated with the 1-D density of states and removed by the energy broadening in the spectral density. Notice that the divergence is mainly due to the phonon emission processes so that the divergence occurs at the LO phonon energy $(=36 \mathrm{meV})$. The major feature associated with the ICFE is, however, that the scattering rates become asymmetric with respect to the direction of the electron motion. This is closely related to the shift of the energy detuning in the spectral density, as already seen in Fig. 1, and can be explained as follows. When an electron moves against the electric field, it gains energy from the electric field during the collision duration with phonons. Therefore, it can emit an LO phonon even if the electron energy is smaller than the threshold energy for phonon emission. In other words, the phonon energy is effectively reduced when the electron moves against the electric field, and the scattering rate shifts to the lower energy-side. On the other hand, an electron loses its energy when it moves along the electric field and, thus, the phonon energy is effec- 
tively increased. As a result, the scattering rate shifts to the higher energy-side.

The transport characteristics could be determined from the quantum kinetic equation described in the previous section. Since the quantum kinetic equation is almost identical to the Boltzmann transport equation, conventional Monte Carlo methods can be applied to numerically solve it by substituting a proper spectral density in the collision integral instead of the energy-conserving delta function.[5] Unfortunately, the spectral density shown in Fig. 1 is not positive definite. Therefore, we approximate it by a Gaussian form with the width and shift given by Eq. (4). The approximated spectral densities employed in the Monte Carlo simulations are plotted by dashed lines in Fig. 1.

Figure 3 shows the electric field dependence of the electron drift velocity at room temperature obtained from the Monte Carlo simulations (dashed line). For comparison, the drift velocity evaluated by the conventional Boltzmann transport equation is also shown (solid line). The dotted line represents the result when only the $\mathrm{CB}$ is included. For the $\mathrm{CB}$, we assume a constant energy-broadening $\left(\Gamma_{k}=2.5 \mathrm{meV}\right)$, as done by Leburton and his group.[6] We would like to stress that the drift velocity is greatly reduced when the

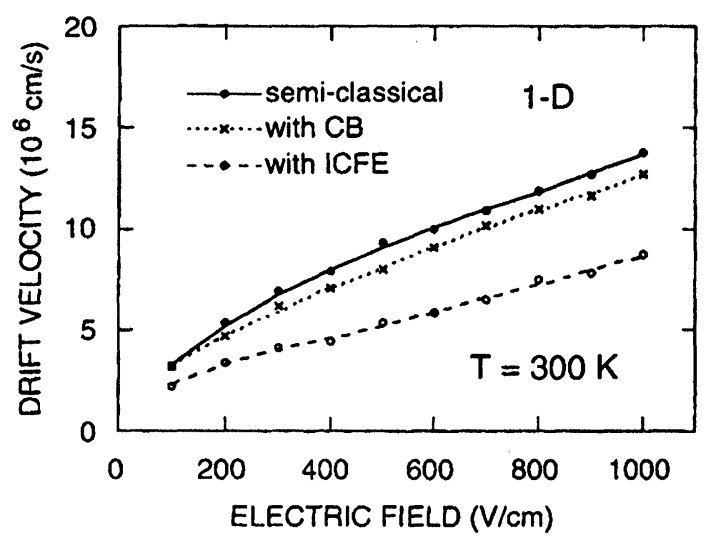

FIGURE 3 Electric field dependence of drift velocity in the ideal 1-D quantum wire. The solid line represents the drift velocity with no quantum correction, whereas the dotted and dashed lines show the respective drift velocities when $\mathrm{CB}$ and ICFE are taken into account
ICFE is included and the reduction becomes greater as the electric field increases. Notice that the CB also reduces the drift velocity, but to a much smaller extent. This implies that the reduction of the drift velocity due to the ICFE is mainly ascribed to the asymmetric phonon scattering rates. Namely, more electrons occupy the states with positive wave-vector because the electric field is directed to the negative direction and, hence, the scattering rate shifted to the lower energy-side (represented by solid lines in Fig. 2 ) is, as a whole, more conducive to electron transport than that shifted to the higher energy-side (represented by dashed lines in Fig. 2). Furthermore, the large peak in the phonon scattering rate indicates the electron transport characteristics is dominantly controlled by electrons with energy below the phononemission threshold energy unless the electric field is extremely strong. Therefore, a slight change in the phonon scattering rates greatly affects the drift velocity and, thus, the ICFE is already significant under moderate electric fields, which is in marked contrast to bulk cases.

\section{CONCLUSIONS}

High-temperature quantum kinetic transport under high electric fields has been investigated with emphasis on the intracollisional field effect (ICFE) in lowdimensional structures. We have shown that the ICFE in GaAs one-dimensional quantum wires is already significant under moderate electric field strengths $(\geq a$ few hundreds $\mathrm{V} / \mathrm{cm}$ ) because of the strong restriction on the electron motion imposed by the low-dimensional structures. Employing the Monte Carlo method including the ICFE, it has been shown that the electron drift velocity in quantum wires could be much smaller than that expected from earlier investigations.

\section{Acknowledgements}

The authors would like to thank Y. Imamura for his ongoing support during the course of this study. One of the authors (N. S.) is also grateful to D. K. Ferry and $\mathrm{K}$. Taniguchi for fruitful conversations. 


\section{References}

[1] For a review, see L. Reggiani, "Recent Results on Hot-Electron Quantum Transport," Physica, vol. 134B, pp. 123 - 131, 1985. Also, see a series of papers by P. Lipavsky et al, "High Field Transport in Semiconductors," Phys. Rev. B, vol. 43, pp. 4885-4896; pp. 6650-6664, pp. 6665-6681, 1991.

[2] J. R. Barker, "High Field Collision Rates in Polar Semiconductors," Solid State Electron., vol. 21, pp. 267- 271, 1978 J. R. Barker and D. K. Ferry, "Self-Scattering Path-Variable Formulation of High-Field, Time-Dependent, Quantum Kinetic Equations for Semiconductor Transport in the FiniteCollision-Duration Regime," Phys. Rev. Lett., vol. 42, pp. 1779-1781, 1979.

[3] C. Jacoboni and L. Reggiani, "The Monte Carlo Method for the Solution of Charge Transport in Semiconductors with Application to Covalent Materials," Rev. Mod. Phys., vol. 55, pp. 645-705, 1983.

[4] N. Sano and A. Yoshii, "Energy Broadening in Ultrafast Relaxation of Photoexcited Electrons: A Monte Carlo Approach," Phys. Rev. B, vol. 48, pp. 1426-1432, 1993.

[5] L. Reggiani, P. Lugli, and A.-P. Jauho, "Monte Carlo Algorithms for Collisional Broadening and Intracollisional Field
Effect in Semiconductor High-Field Transport," J. Appl. Phys., vol. 64, pp. 3072-3078, 1988.

[6] For a recent review, see J. P. Leburton and D. Jovanovic, "Hot-Carrier Dynamics in Mesoscopic Structures," Semicond. Sci. Technol., vol. 7, pp. B202-B209, 1992. Also, see N. Mori and C. Hamaguchi, "Hot-Electron Transport in Quantum Structures," Semicond. Sci. Technol., vol. 9, pp. 941-945, 1994.

\section{Biographies}

Nobuyuki Sano is with NTT LSI Laboratories, Kanagawa, Japan. He is interested in device physics with emphasis on high-field carrier transport in semiconductors.

Akira Yoshii is a Group Leader of Device Physics Group at NTT LSI Laboratories, Kanagawa, Japan. His interests include device simulation, device modeling, and device physics including quantum effects. 

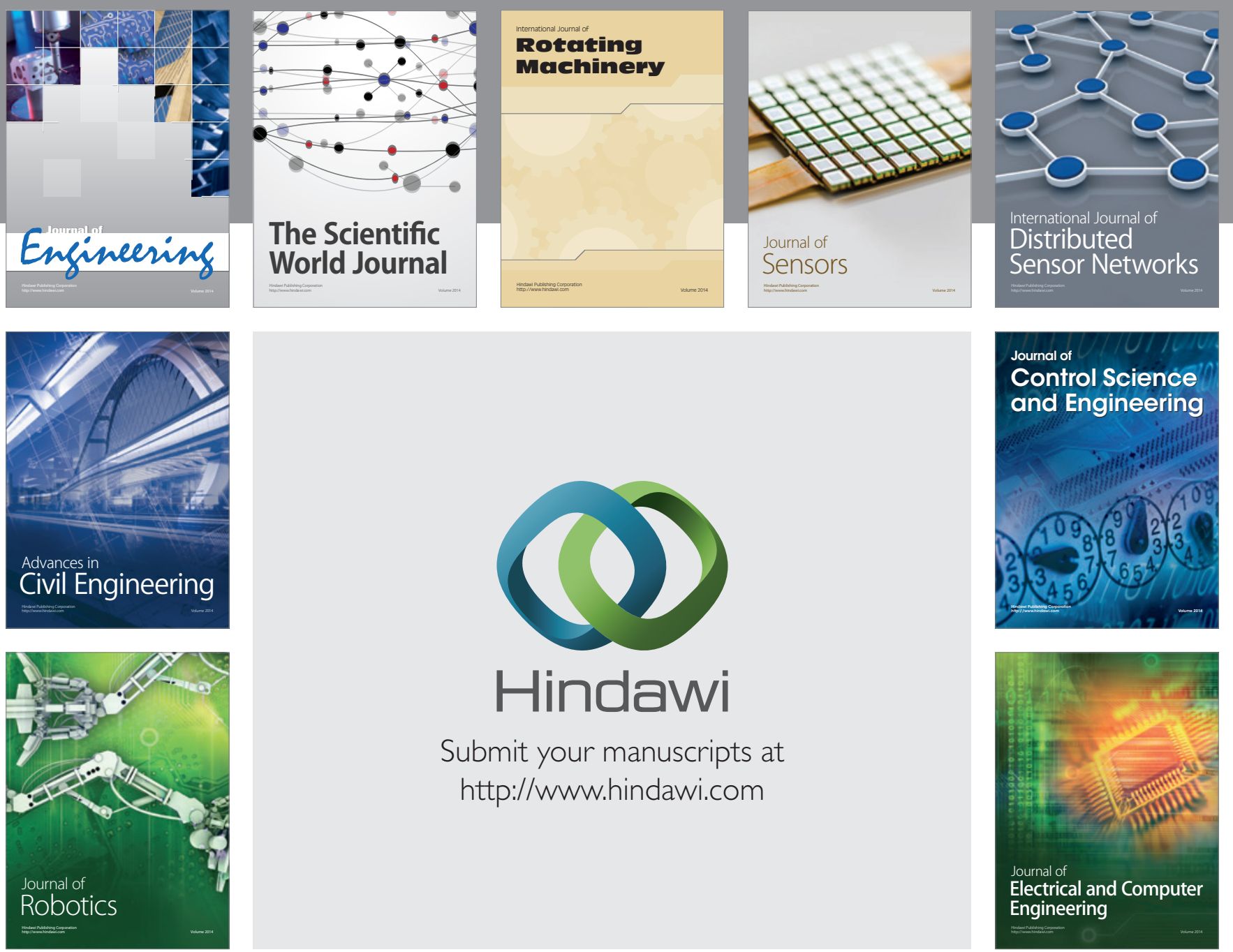

Submit your manuscripts at

http://www.hindawi.com
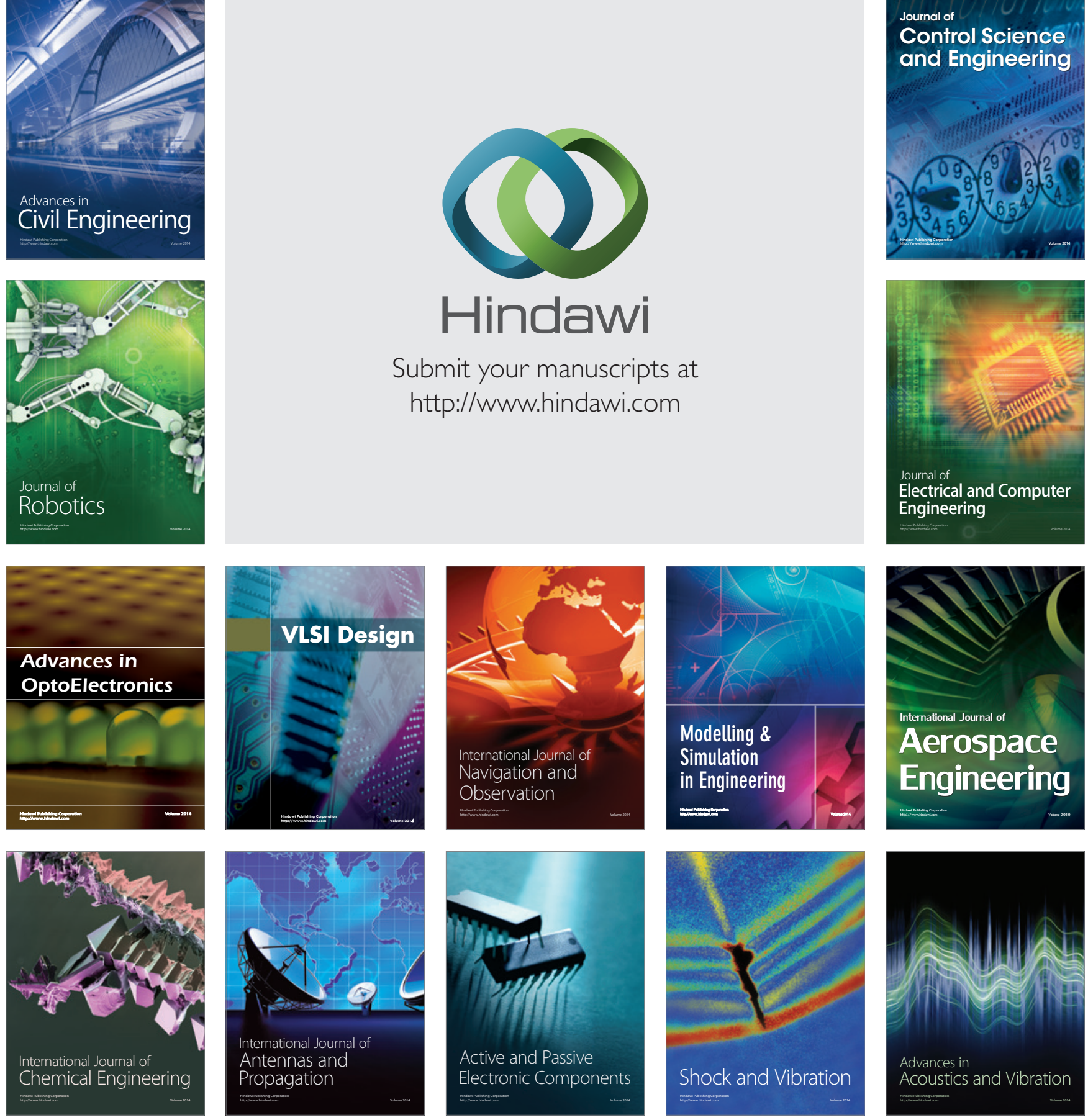